\title{
Aneuploidy in neuroblastoma tumors is not associated with inactivating point mutations in the STAG2 gene
}

\author{
Anna Djos ${ }^{1}$, Susanne Fransson ${ }^{1}$, Per Kogner ${ }^{2}$ and Tommy Martinsson ${ }^{1^{*}}$
}

\begin{abstract}
Background: Chromosomal instability is a hallmark of human cancer caused by errors in mitotic control and chromosome segregation. STAG2 encodes a subunit of the cohesion complex that participates in mitotic chromatid separation and was recently found to show low expression and inactivating mutations in Ewing's sarcoma, melanoma and glioblastoma.

In the childhood tumor neuroblastoma (NB) segmental chromosomal alterations are associated with poor prognosis whereas tumors displaying whole chromosome gains and losses have a much better prognosis.
\end{abstract}

Method: As the genetic contribution to aneuploidy is unknown in NB, we investigated the presence of STAG2 mutations through sequence analysis of all 33 coding exons in 37 primary NB tumors.

Results and conclusion: As no STAG2 mutation was detected in this study, we conclude that inactivating mutation of STAG2 is not likely causative to neuroblastoma aneuploidy.

Keywords: Neuroblastoma, STAG2, Aneuploidy, Numerical aberrations, Chromosomal instability

\section{Background}

Chromosome instability leading to numerical and segmental alteration is a hallmark of human cancer genomes, contributing to malignant transformation. Whereas segmental rearrangements directly can promote cancer through activating oncogenes and inactivating tumors suppressor genes, aneuploidy could provide a tumor driving function through dosage-dependent mechanisms [1]. Aneuploidy is caused by inaccurate chromosome segregation, possibly due to genetic alteration of various participants of the segregation or mitotic checkpoint machinery. Recently, Solomon et al., showed the presence of inactivating mutations in the STAG2 gene located at Xq25 in primary tumors and cancer cell lines originating from glioblastoma, melanoma and Ewing's sarcoma. The STAG2 mutations reported by Solomon et al., represented an array of different alterations (missense-, nonsense-, splice site mutations as well as intragenic deletions), mainly concentrated to

\footnotetext{
* Correspondence: tommy.martinsson@gu.se

'Department of Clinical Genetics, Institute of Biomedicine, University of Gothenburg, Sahlgrenska University Hospital, SE-413 45 Gothenburg, Sweden Full list of author information is available at the end of the article
}

hotspot regions corresponding to exon 9, 11, 12 and 20 [2]. STAG2 encodes a subunit of the multimeric cohesion complex required in regulating sister chromatid separation during mitosis [3-5]. Inactivation of STAG2 in cell lines with stable karyotype resulted in increased number of abnormal mitotic events (e.g. lagging chromosomes and anaphase bridges) ultimately leading to increased aneuploidy rate while mutational correction of endogenous STAG2 mutations in aneuploid cell lines resulted in enhanced chromosomal stability. Further, loss of STAG2 protein was observed in several human cancer cell lines, primary tumors and xenografts of different origin [2].

Neuroblastoma (NB), a childhood cancer of the postganglionic nervous system show broad biological and clinical heterogeneity, ranging from highly aggressive tumors with fatal outcome to tumors with spontaneous regression. Advanced disease and poor prognosis in NB patients is commonly associated with either $M Y C N$ amplification or 11q-deletion, representing two separate NB subgroups with different genomic profiles. Whereas $M Y C N$ amplified tumors commonly have few segmental or numerical aberrations besides $17 \mathrm{q}$-gain and $1 \mathrm{p}$-deletions, $11 \mathrm{q}$-deleted NB generally contains many more segmental alterations. In 
contrast, NB tumors with numerical aberrations (i.e. only whole chromosome gains and/or losses) without other segmental alterations instead have excellent outcome [6] and the underlying mechanisms causing aneuploidy in NB tumors are yet unknown.
As the findings of Solomon et al [2] indicate that loss of functional STAG2 protein could contribute to increased chromosomal instability we wanted to investigate the presence of inactivating mutations in STAG2 in prognostically favorable NB with common aneuploidy

Table 1 Clinical and biological data of the tumors

\begin{tabular}{|c|c|c|c|c|c|c|c|c|c|c|c|}
\hline Sample & INSS & INRG & Gender & 1p-del & MNA & 11q-del & 17q-gain & Genomic profile* & 5-year OS & Sanger & NGS \\
\hline $3 \mathrm{E} 2$ & 4 & $M$ & $\mathrm{Ma}$ & neg & neg & pos & pos & 11q-del & DOD & & + \\
\hline $4 \mathrm{E} 1$ & 4 & $M$ & $\mathrm{~F}$ & neg & neg & pos & pos & $11 \mathrm{q}-\mathrm{del}$ & DOD & & + \\
\hline $5 \mathrm{E} 1$ & $2 \mathrm{~A}$ & $L$ & $\mathrm{~F}$ & neg & neg & neg & neg & Num only & NED & + & \\
\hline $6 \mathrm{E} 9$ & 3 & $L$ & $\mathrm{Ma}$ & pos & neg & pos & pos & 11q-del & DOD & & + \\
\hline $8 \mathrm{E} 4$ & 1 & $\mathrm{~L}$ & $\mathrm{~F}$ & neg & neg & neg & neg & Num only & NED & + & \\
\hline $8 \mathrm{E} 5$ & 3 & $L$ & $\mathrm{~F}$ & neg & neg & neg & neg & Num only & NED & + & \\
\hline $11 \mathrm{E} 1$ & 4 & $M$ & $\mathrm{~F}$ & neg & neg & pos & pos & 11q-del & NED & & + \\
\hline $11 \mathrm{E} 4$ & 4 & $M$ & $F$ & neg & neg & pos & pos & 11q-del & DOD & & + \\
\hline $11 \mathrm{E} 8$ & 1 & $L$ & $\mathrm{Ma}$ & neg & neg & neg & neg & Num only & NED & + & + \\
\hline $12 \mathrm{E} 5$ & $4 S$ & MS & $\mathrm{Ma}$ & neg & neg & neg & neg & Num only & NED & + & \\
\hline $13 \mathrm{E7}$ & $4 S$ & MS & $\mathrm{F}$ & neg & neg & neg & neg & Num only & NED & + & \\
\hline $15 E 7$ & 3 & $\mathrm{~L}$ & $\mathrm{Ma}$ & neg & neg & neg & neg & Num only & DSC & + & \\
\hline $16 \mathrm{E} 1$ & 1 & $L$ & $\mathrm{~F}$ & neg & neg & neg & neg & Num only & NED & + & \\
\hline $16 \mathrm{E} 2$ & $4 S$ & $L$ & $\mathrm{Ma}$ & neg & neg & neg & neg & Num only & NED & + & \\
\hline 17E5 & 4 & $M$ & $\mathrm{~F}$ & neg & neg & neg & neg & Num only & DOD & + & \\
\hline $18 \mathrm{E} 2$ & 1 & $L$ & $\mathrm{~F}$ & neg & neg & pos & pos & $11 \mathrm{q}$-del & NED & & + \\
\hline $18 \mathrm{E} 5$ & 1 & $L$ & $\mathrm{~F}$ & neg & neg & neg & neg & Num only & NED & + & \\
\hline 9R9 & 3 & M & $\mathrm{F}$ & pos & neg & pos & pos & 11q-del & DOD & & + \\
\hline 10R6 & $2 \mathrm{~A}$ & $\mathrm{~L}$ & $\mathrm{Ma}$ & neg & neg & neg & neg & Num only & NED & + & \\
\hline 10R8 & 3 & L & $\mathrm{F}$ & neg & neg & pos & neg & Num only & DOD & & + \\
\hline 13R1 & 3 & $L$ & $\mathrm{Ma}$ & pos & pos & neg & pos & MNA & DOD & & + \\
\hline 15R8 & 3 & $M$ & Ma & pos & neg & pos & pos & 11q-del & NED & & + \\
\hline $23 R 4$ & 2 & $L$ & $\mathrm{~F}$ & neg & neg & neg & neg & Num only & NED & + & \\
\hline $25 R 6$ & 4 & $M$ & $\mathrm{Ma}$ & pos & pos & neg & pos & MNA & DOD & & + \\
\hline $25 R 7$ & 1 & $L$ & $\mathrm{Ma}$ & pos & neg & neg & neg & Other segm. & NED & + & \\
\hline $26 \mathrm{R} 1$ & 1 & $L$ & $\mathrm{Ma}$ & neg & neg & neg & neg & Num only & NED & + & \\
\hline $27 R 1$ & $2 \mathrm{~A}$ & $L$ & $\mathrm{~F}$ & neg & neg & neg & neg & Num only & NED & + & \\
\hline $27 R 7$ & 2 & $L$ & $\mathrm{Ma}$ & neg & neg & neg & neg & Num only & NED & + & \\
\hline $28 R 8$ & 4 & M & $\mathrm{Ma}$ & neg & neg & pos & pos & 11q-del & DOD & & + \\
\hline $30 \mathrm{RO}$ & 4 & $M$ & $\mathrm{~F}$ & neg & neg & pos & neg & 11q-del & NED & & + \\
\hline $34 \mathrm{R} 5$ & 3 & $L$ & $\mathrm{Ma}$ & neg & neg & neg & neg & Num only & NED & + & \\
\hline 37R5 & 1 & $\mathrm{~L}$ & $\mathrm{Ma}$ & neg & neg & neg & neg & Num only & NED & + & \\
\hline $44 \mathrm{R} 5$ & 1 & $\mathrm{~L}$ & $\mathrm{~F}$ & neg & neg & neg & neg & Num only & NED & & + \\
\hline $46 R 6$ & 4 & $M$ & $\mathrm{Ma}$ & neg & neg & pos & pos & 11q-del & NED & & + \\
\hline 47R4 & 4 & $M$ & $\mathrm{~F}$ & neg & neg & pos & pos & $11 \mathrm{q}$-del & NED & & + \\
\hline 49R1 & 4 & M & $\mathrm{Ma}$ & neg & neg & pos & pos & 11q-del & NED & & + \\
\hline $52 \mathrm{R} 2$ & 1 & $L$ & $\mathrm{Ma}$ & neg & neg & neg & pos & 17q-gain & NED & & + \\
\hline
\end{tabular}

Abbreviations: INSS International Neuroblastoma Staging System [7], INRG International Neuroblastoma Risk Group [8], M metastatic, L localized, Ma male, $F$ female, neg negative, pos positive, MNA NMYC-amplification, NED no evidence of disease, DOD dead of disease, DSC dead of surgical complications. *As defined by Carén et al [6]. 
pattern and prognostically unfavorable NB with high degree of segmental alteration.

\section{Methods}

\section{Cell material}

Genomic DNA of 37 primary NB tumors and cell lines was extracted with DNeasy blood and tissue kit (Qiagen, Hilden Germany) according to the manufacturer's instructions. Mutation analysis through Sanger sequencing of the 33 coding exons (3-35) of STAG2 was performed on 18 favorable NB tumor samples (stage 1, 2 and 4 S), whereas additional 19 tumors of different risk groups were subjects for whole exome sequencing whereof one was also evaluated through Sanger sequencing (Table 1). In addition, mutation analysis with Sanger sequencing was performed on eleven NB cell lines (Table 2) SK-N-AS, SK-N-BE(2), SK-N-F1, SH-SY-5Y, SK-N-SH, SK-N-DZ, NB69, Kelly, IMR32, LS and NGP) for exons 3, 9, 11, 12, 20, 23 and 25. Informed consent was obtained from the parents of the patients. Ethical permission was granted by the ethics committee (Karolinska Institutet and Karolinska University Hospital, registration number 03-736 and 09-1369).

\section{High resolution SNP array analysis}

Genomic profiling of the 37 primary NB tumors (Table 1, Figure 1) were performed with Genechip Human Mapping 250K NspI (Affymetrix Inc., Santa Clara, CA) according to the experimental procedure previously described [9].

\section{Sanger sequencing}

Sanger sequencing was performed as earlier described [10] and primers were designed manually or with the use of ExonPrimer (http://ihg.gsf.de/ihg/ExonPrimer.html), primer sequences are available upon request. The sequence data were analyzed using Seqscape v2.5 (Applied Biosystems).

Table 2 Characteristics of the cell lines

\begin{tabular}{lllllll}
\hline Cell line & Gender & 1p-del & MNA & 11q-del & 17q-gain & ChrX \\
\hline NGP & Ma & neg & pos & pos & pos & one \\
Kelly & $F$ & neg & pos & pos & pos & one \\
LS & $F$ & neg & pos & neg & pos & two \\
SH-SY-5Y & $F$ & neg & neg & neg & pos & two \\
NB69 & Ma & pos & neg & neg & pos & one \\
SK-N-FI & Ma & neg & neg & neg & pos & one \\
SK-N-AS & $F$ & pos & neg & pos & pos & one \\
IMR32 & Ma & pos & pos & neg & pos & one \\
SK-N-BE & Ma & neg & pos & neg & pos & one \\
SK-N-DZ & $F$ & neg & pos & pos & neg & two \\
SK-N-SH & $F$ & neg & neg & neg & pos & two \\
\hline
\end{tabular}

Abbreviations: $M a$ male, $F$ female, neg negative, pos positive.

\section{Exome sequencing}

Whole exome capturing was made with $5 \mu \mathrm{g}$ gDNA using SureSelect Human All Exon Target Enrichment System 3.0 (Agilent Technologies, Santa Clara, CA) or TrueSelect exome enrichment kit (Illumina, San Diego, CA) before pair-end sequencing on Illumina HiScanSQ or HighSeq2000. Alignment to the hg19 reference genome (http://genome.ucsc.edu/) was performed using BWA [11], indel realignment with GATK [12] while functional variant annotation was made through ANNOVAR [13]. Visualisation for manual reviewing of alignments was performed using the DNAnexus software (DNAnexus Inc. Mountain View, CA) or the Integrative Genomic Viewer [14].

\section{Results}

The coding sequence of the STAG2 gene was screened with Sanger sequencing and targeted re-sequencing in a panel of 37 NB tumors and eleven NB cell lines (Tables 1 and 2). High-quality sequence of the STAG2 gene was obtained for all 33 coding exons both from Sanger- and exome sequencing. Sequences with an average coverage of 51x (range 13-117x) were acquired from all coding exons in tumors analyzed by exome sequencing.

One of 37 primary tumors (11E8) and one of eleven NB cell lines (SK-N-DZ) showed a hemizygous and heterozygous base substitution respectively corresponding to $\mathrm{C}>\mathrm{T}$ at mRNA position 1298-9 in intron 10 (Figure 2), similar to a finding earlier reported in a glioblastoma primary xenograft [2]. However, this variant corresponding to SNP rs7063522, was also present in normal DNA from patient's blood (Figure 2). No exonic variants were detected in our set of $37 \mathrm{NB}$ tumors and 11 NB cell lines, neither in the hot spot positions reported by Solomon et al., nor in any other coding region of the STAG2 gene [2].

\section{Discussion}

Aneuploidy results from missegregation of sister chromatids during mitotic cell division. In NB segmental chromosomal alterations are common in aggressive tumors whereas tumors with only whole chromosome gains and losses are prognostically more favorable [6]. However, the cause of the high aneuploidy rate in this favorable group of NB tumors is currently unknown. Aneuploidy is also frequently observed in other malignancies and recently STAG2 was recognized as a tumor suppressor gene causing malfunctioning mitotic sister chromatid separation when inactivated. Inactivating mutations of STAG2 have been found in glioblastoma, Ewing's sarcoma and melanoma, possibly contributing to chromosome instability in these tumors [2] and this led us to investigate whether they might also be responsible for the high aneuploidy rate seen in favorable NB tumors. 


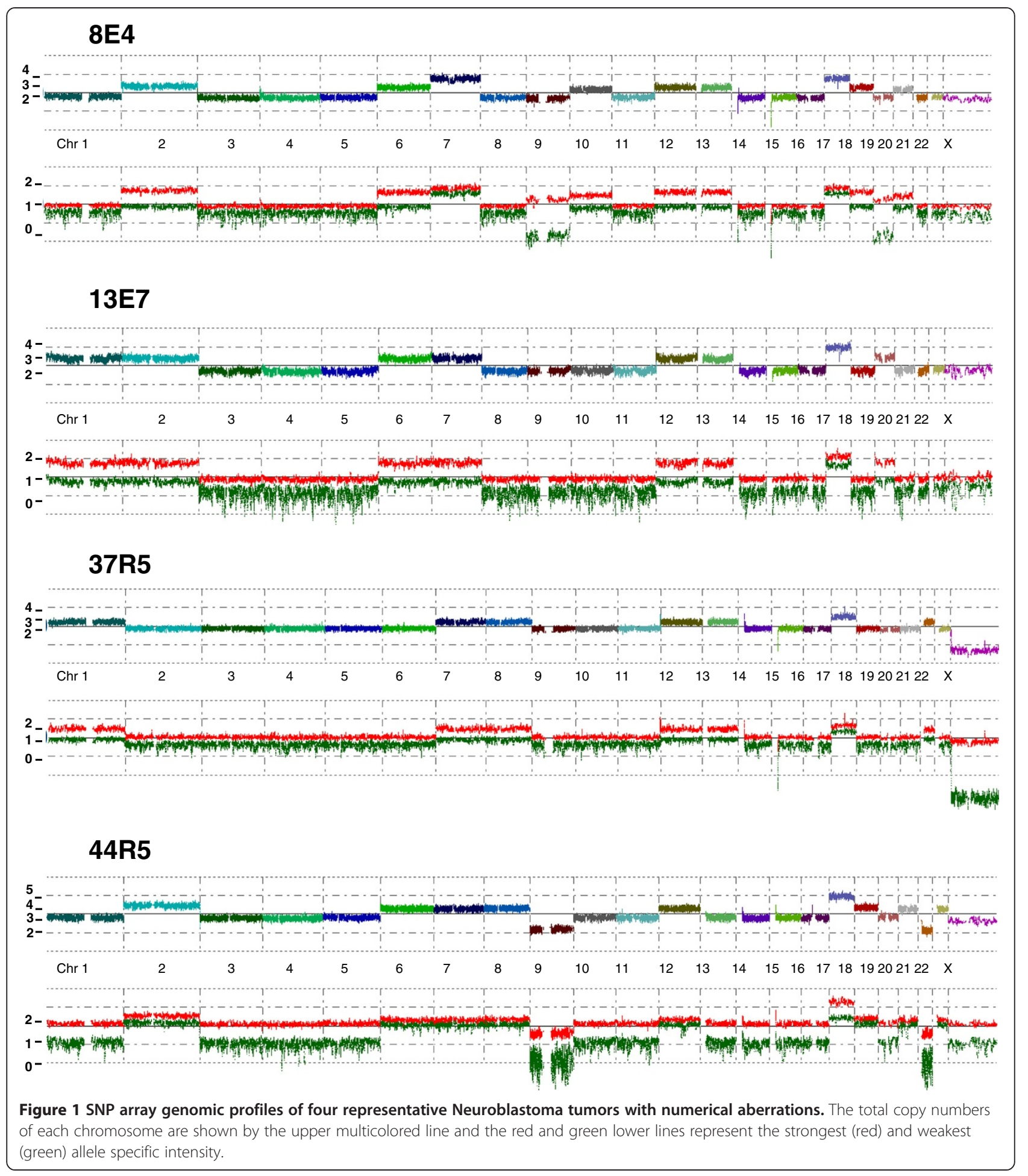

In the present study, we sequenced all 33 coding exons of the STAG2 gene in $11 \mathrm{NB}$ cell lines and 37 primary NB tumors and no deviation from the human reference sequence could be detected. This is similar to other recent studies of the STAG2 mutational hot spot region (corresponding to exon 9, 11, 12 and 20) in 90 acute leukemia samples [15] and 225 adult carcinomas of various origin (colorectal-, gastric-, breast-, prostate carcinoma and non-small lung cell cancer) [16]. Alteration of STAG2 was not detected in either study, suggesting that 


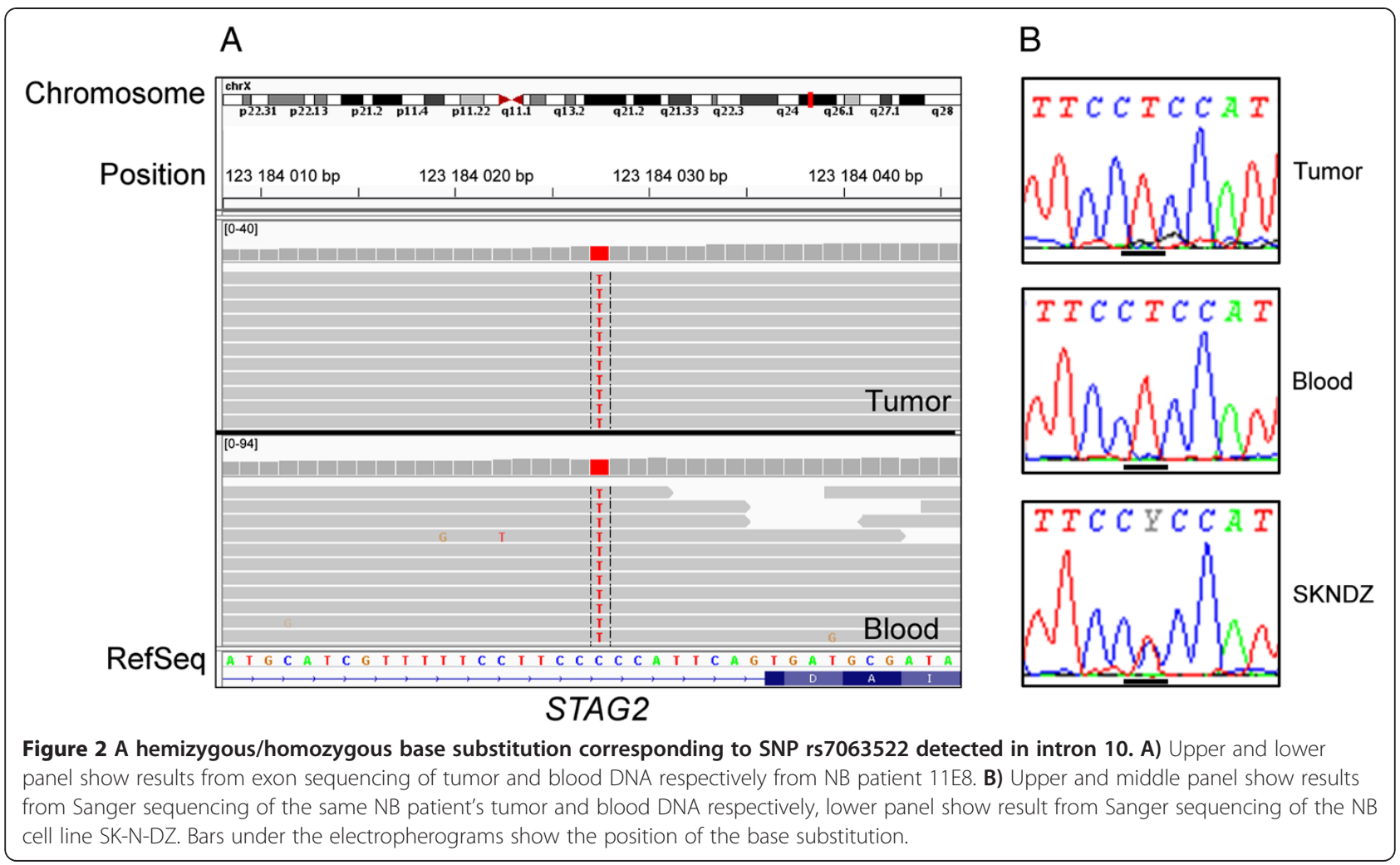

STAG2 inactivation is not due to inactivating gene mutations in these malignancies. Other gene silencing mechanisms might contribute as STAG2 protein was lost in $23-30 \%$ of studied carcinomas [16].

According to the publicly accessible R2-microarray analysis and visualization platform (http://r2.amc.nl), STAG2 expression in NB can be correlated with outcome in that lower STAG2 expression is correlated to lower overall survival and relapse free survival. Other causes than STAG2 mutations are likely to be involved in this, e.g. epigenetic events.

Furthermore, previous and subsequent analyzes of copy number variations performed by us in totally 360 primary NB tumors show that segmental deletions of the chromosomal region Xq25 harboring STAG2 are not seen ([6] and unpublished data).

Taken together these data suggest that STAG2 mutations are not likely responsible for the high aneuploidy rate in NB tumors with numerical aberrations. However, other genes participating in the mitotic checkpoint control may be responsible for the chromosomal missegregation in NB tumors with many numerical aberrations.

Three major next generation sequencing studies have together analyzed a large serie of NB tumors [17-19]. No STAG2 mutations are reported and overall very few recurrent amino-acid changing mutations were found. Molenaar et al. [17], found few recurrent mutations except for the genes $A L K$ and TIAM1 and mutations in genes regulating neuritogenes. Cheung et al. [18], reported the finding of deletions and loss-of-function mutations in the ATRX gene and the association with advanced age at NB diagnosis. Furthermore, Sausen et al. [19], found mutations and deletions in the genes ARID1A and ARID1B which were associated with treatment failure and poor prognosis. Collectively more recurrent somatic mutation have been found in high risk NB patients compared to patients with stage 1, 2 and $4 \mathrm{~S}$ tumors and in the published NGS data we cannot identify clear candidate genes that could be responsible for the high aneuploidy rate seen in favorable NB.

\section{Conclusion}

Here we show that inactivating point mutations in the STAG2 gene are not common in NB tumors and that aneuploidy seen in NB tumors is likely due to other participants of the mitotic checkpoint and chromosome segregation machinery.

\section{Competing interests}

The authors declare they have no conflict of interests.

\section{Authors' contributions}

AD and SF performed experiments, analysis and wrote the paper. TM coordinated the project, critically reviewed the manuscript and provided biological and clinical information together with PK. All authors read and approved the final manuscript. 


\section{Acknowledgements}

We would like to thank the Genomics and Bioinformatics core facilities at University of Gothenburg and the SciLifeLab, Stockholm for access to instruments. We also thank Rose-Marie Sjöberg for excellent technical assistance. This work was supported by grants from the Swedish Cancer Society, the Swedish Children's Cancer Foundation, the Sahlgrenska University Hospital Foundation, the Swedish state through the LUA/ALF agreement, and the Assar Gabrielsson Foundation.

\section{Author details}

'Department of Clinical Genetics, Institute of Biomedicine, University of Gothenburg, Sahlgrenska University Hospital, SE-413 45 Gothenburg, Sweden. ${ }^{2}$ Childhood Cancer Research Unit, Department of Woman and Child Health, Karolinska Institute, Karolinska University Hospital, SE-17176 Stockholm, Sweden.

Received: 4 March 2013 Accepted: 23 September 2013 Published: 2 October 2013

\section{References}

1. Gordon DJ, Resio B, Pellman D: Causes and consequences of aneuploidy in cancer. Nat Rev Genet 2012, 13(3):189-203.

2. Solomon DA, Kim T, Diaz-Martinez LA, Fair J, Elkahloun AG, Harris BT, Toretsky JA, Rosenberg SA, Shukla N, Ladanyi M, et al: Mutationa inactivation of STAG2 causes aneuploidy in human cancer. Science 2011, 333(6045):1039-1043.

3. Carramolino L, Lee BC, Zaballos A, Peled A, Barthelemy I, Shav-Tal Y, Prieto I, Carmi P, Gothelf Y, Gonzalez De Buitrago G, et al: SA-1, a nuclear protein encoded by one member of a novel gene family: molecular cloning and detection in hemopoietic organs. Gene 1997, 195(2):151-159.

4. Sumara I, Vorlaufer E, Gieffers C, Peters BH, Peters JM: Characterization of vertebrate cohesin complexes and their regulation in prophase. J Cell Biol 2000, 151(4):749-762.

5. Haering $\mathrm{CH}$, Farcas AM, Arumugam P, Metson J, Nasmyth K: The cohesin ring concatenates sister DNA molecules. Nature 2008, 454(7202):297-301.

6. Caren H, Kryh H, Nethander M, Sjoberg RM, Trager C, Nilsson S, Abrahamsson J, Kogner P, Martinsson T: High-risk neuroblastoma tumors with 11q-deletion display a poor prognostic, chromosome instability phenotype with later onset. Proc Natl Acad Sci USA 2010, 107(9):4323-4328.

7. Brodeur GM, Pritchard J, Berthold F, Carlsen NL, Castel V, Castelberry RP, De Bernardi B, Evans AE, Favrot M, Hedborg F, et al: Revisions of the international criteria for neuroblastoma diagnosis, staging, and response to treatment. J Clin Oncol 1993, 11(8):1466-77.

8. Monclair T, Brodeur GM, Ambros PF, Brisse HJ, Cecchetto G, Holmes K, Kaneko M, London WB, Matthay KK, Nuchtern JG, et al: The International Neuroblastoma Risk Group (INRG) staging system: an INRG Task Force report. J Clin Oncol 2009, 27:298-303.

9. Caren H, Erichsen J, Olsson L, Enerback C, Sjoberg RM, Abrahamsson J, Kogner $\mathrm{P}$, Martinsson T: High-resolution array copy number analyses for detection of deletion, gain, amplification and copy-neutral LOH in primary neuroblastoma tumors: four cases of homozygous deletions of the CDKN2A gene. BMC Genomics 2008, 9:353.

10. Caren $H$, Abel F, Kogner $P$, Martinsson T: High incidence of DNA mutations and gene amplifications of the ALK gene in advanced sporadic neuroblastoma tumours. Biochem J 2008, 416(2):153-159.

11. Li H, Durbin R: Fast and accurate short read alignment with BurrowsWheeler transform. Bioinformatics 2009, 25(14):1754-1760.

12. McKenna A, Hanna M, Banks E, Sivachenko A, Cibulskis K, Kernytsky A, Garimella K, Altshuler D, Gabriel S, Daly M, et al: The Genome Analysis Toolkit: a MapReduce framework for analyzing next-generation DNA sequencing data. Genome Res 2010, 20(9):1297-1303.

13. Wang K, Li M, Hakonarson H: ANNOVAR: functional annotation of genetic variants from high-throughput sequencing data. Nucleic Acids Res 2010, 38(16):e164.

14. Thorvaldsdottir H, Robinson JT, Mesirov JP: Integrative Genomics Viewer (IGV): high-performance genomics data visualization and exploration. Brief Bioinform 2013, 4(2):178-92.

15. Chung NG, Kim MS, Yoo NJ, Lee SH: Somatic mutation of STAG2, an aneuploidy-related gene, is rare in acute leukemias. Leuk Lymphoma 2012, 53(6):1234-1235.
16. Kim MS, Kim SS, Je EM, Yoo NJ, Lee SH: Mutational and expressional analyses of STAG2 gene in solid cancers. Neoplasma 2012, 59(5):524-529.

17. Molenaar JJ, Koster J, Zwijnenburg DA, van Sluis P, Valentijn $L J$, van der Ploeg I, Hamdi M, van Nes J, Westerman BA, van Arkel J, et al: Sequencing of neuroblastoma identifies chromothripsis and defects in neuritogenesis genes. Nature 2012, 483(7391):589-93.

18. Cheung NK, Zhang J, Lu C, Parker M, Bahrami A, Tickoo SK, Heguy A, Pappo AS, Federico S, Dalton J, et al: Association of age at diagnosis and genetic mutations in patients with neuroblastoma. JAMA 2012, 307(10):1062-71. doi:10.1001/jama.2012.228.

19. Sausen M, Leary RJ, Jones S, Wu J, Reynolds CP, Liu X, Blackford A, Parmigiani G, Diaz LA Jr, Papadopoulos $N$, et al: Integrated genomic analyses identify ARID1A and ARID1B alterations in the childhood cancer neuroblastoma. Nat Genet 2013, 45(1):12-7.

doi:10.1186/1471-2350-14-102

Cite this article as: Djos et al.: Aneuploidy in neuroblastoma tumors is not associated with inactivating point mutations in the STAG2 gene. BMC Medical Genetics 2013 14:102.

\section{Submit your next manuscript to BioMed Central and take full advantage of:}

- Convenient online submission

- Thorough peer review

- No space constraints or color figure charges

- Immediate publication on acceptance

- Inclusion in PubMed, CAS, Scopus and Google Scholar

- Research which is freely available for redistribution

Submit your manuscript at www.biomedcentral.com/submit
C Biomed Central 